\title{
BioSpotlight
}

\section{In living color}

The Lowry assay is the most commonly used and cited method for quantifying protein content from a biological sample. While the method is simple, sensitive, and precise, for samples containing material that adversely react with Folin's reagent, an essential component of the Lowry assay, accurate measurements are difficult or even impossible. This can be overcome in many cases by using an appropriate blank for absorbance readings, but lipids, detergents, and colored substances remain a source of interference for the Lowry assay. A modified Lowry assay, U-1988, allowed researchers to accurately measure proteins in the presence of lipids and detergents, but colored compounds, such as those found in fruits and vegetables, pigmented microbes, or bile, still required extensive chromatography for analysis. To provide an alternative to cumbersome chromatography methods, Upreti et al. from the Plant and Food Research Ruakura in Hamilton, New Zealand further modified the U-1988 Lowry assay to improve convenience, enable measurements of protein content in colorless and colored biological samples, and accurately measure proteins even at low concentrations with improved sensitivity. The authors replaced the unstable carbonate buffer used in previous Lowry assays with a phosphate buffer, which not only increased the shelf life of the reagent (providing room temperature stability for two weeks compared to the need for daily preparation of the carbonate buffer), but also improved assay sensitivity by $25 \%$. In addition, interference with absorbance readings caused by bubbles was reduced by incorporating acetonitrile into the assay reagents. But the greatest enhancement of the new Lowry assay is found in a decolorizing protocol that is compatible with the downstream colorimetric protein assay, allowing for protein quantification of colored samples. Selective precipitation of proteins was accomplished using perchloric acid, while pigment interference was avoided by oxidation with hydrogen peroxide at $50^{\circ} \mathrm{C}$. Addition of pyruvate following decolorization of the samples destroyed any remaining hydrogen peroxide, thus preventing deterioration of the colorimetric signal needed for protein quantification. The details of the new procedure, which the authors have named U-2012, and its demonstration by quantifying proteins in red wine, beetroot, and blueberry are presented in the current issue of BioTechniques.

See "U-2012: An improved Lowry protein assay, insensitive to sample color, offering reagent stability and enhanced sensitivity" on page 159.

\section{The IRES finally audits worm researchers}

A key technique in $C$. elegans molecular biology is the generation of transgenic lines expressing a gene of interest. This is typically accomplished through the co-microinjection of two constructs one expressing the gene of interest and the other containing a marker gene - into the syncytial gonad. The two genes then become incorporated into large, heritable extrachromosomal arrays, such that transformants can be selected or identified by expression of the marker gene. Although a well-established approach to generate transgenic animals, there are disadvantages to this method, including the possibility of interference by the marker construct with the proper expression of the gene of interest. As a result, single selectable transformation vectors are often preferable, but these commonly require the fusion of the gene of interest with a reporter gene such as GFP, potentially affecting the structure or function of the target protein. Ideally, a gene of interest and a reporter gene in a single transformation vector would be translated separately from a bicistronic mRNA containing an internal ribosome entry site (IRES). Unlike other organisms such as viruses, bacteria, yeast,

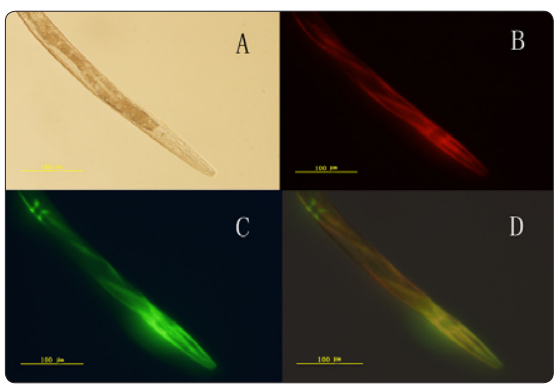

Micrographs of a $C$. elegans transformed with the myo-3 promoter-RFP-IRES-GFP construct. A. Brightfield. B. RFP fluorescence expression. C. GFP fluorescence expression. D. Merged view of panels $B$ and $C$..

and mammalian cells, however, an IRES sequence had not been previously identified in C. elegans. In this issue, Li and Wang, at the China Agriculture University (Beijing, China), report their isolation of an IRES sequence from $C$. elegans. Through bioinformatics analysis, the authors identified a sequence upstream of the $H s p 3$ gene that possessed the Y-shaped double-hairpin secondary structure characteristic of IRES elements. This putative IRES sequence was then tested in a bicistronic expression construct consisting of the myo-3 promoter, RFP, the putative IRES, and GFP for its ability to initiate translation of the second open reading frame (i.e. GFP) in the bicis- tronic mRNA. Transgenic worms with this construct clearly showed expression of both RFP and GFP in muscle tissue, with expression of both proteins further confirmed by Western blots. Control constructs verified that the $C$. elegans IRES sequence was required for GFP expression, and that the element was not acting as a cryptic promoter for GFP expression. This newly identified IRES sequence will enable the construction of bicistronic transformation vectors that should prove to be powerful new tools for $C$. elegans molecular biology.

See "Construction of a bicistronic vector for the co-expression of two genes in Caenorhabditis elegans using a newly identified IRES” on page 173.

Written by Patrick C.H. Lo, Ph.D. and Kristie Nybo, Ph.D. Ifi

BioTechniques 52:125 (March 2012)

doi 10.2144/000113813

To purchase reprints of this article, contact:

biotechniques@fosterprinting.com 